\title{
MEDICAL IMAGE SEGMENTATION
}

\author{
H.S.Prasantha ${ }^{1}$ Dr.Shashidhara.H.L ${ }^{2}$ Dr.K.N.B.Murthy ${ }^{3}$ Madhavi Lata.G ${ }^{4}$ \\ PES Institute of Technology, Bangalore, Karnataka, India \\ ${ }^{1}$ Prashanth 34@rediffmail.com 2 shashihl@yahoo.com \\ ${ }^{3}$ principal@pes.edu ${ }^{4}$ madhu.g.86@gmail.com
}

\begin{abstract}
Image segmentation is an essential but critical component in low level vision image analysis, pattern recognition, and in robotic systems. It is one of the most difficult and challenging tasks in image processing which determines the quality of the final result of the image analysis. Image segmentation is the process of dividing an image into different regions such that each region is homogeneous. Various image segmentation algorithms are discussed. Some examples in different image formats are presented and overall results discussed and compared considering different parameters.
\end{abstract}

Keywords: Segmentation; Thresholding; Clustering; Watershed; Genetic; Histogram, Region based

\section{INTRODUCTION}

The applications of image processing include scaling, segmentation, compression, enhancement, detection, feature extraction, restoration etc. Segmentation of an image is the division or separation of the image into regions of similar attribute. The most basic attribute for segmentation is image luminance for monochrome image and color components for the color image. Segmentation is required to distinguish objects from background. Some of the practical applications of image segmentation include Medical imaging such as locating tumors and other pathologies, measuring tissue volumes, locating objects in satellite images, face recognition, Traffic control systems, Fingerprint recognition and Machine vision etc.

\section{IMAGE SEGMENTATION ALGORITHMS}

Image segmentation is the process of assigning a label to every pixel in an image such that pixels with the same label share certain visual characteristics. The different Image segmentation algorithms are Feature-Space Based Techniques, Clustering (K-means algorithm \& Fuzzy k-means algorithm), Histogram thresholding, Image-Domain or Region Based Techniques (Split-and-merge techniques, Region growing techniques, Neural-network based techniques, Edge Detection Technique), Fuzzy Techniques and Physics Based Approaches

\section{A. Histogram Thresholding}

Histogram thresholding is one of the widely used techniques for monochrome image segmentation. It assumes that the images are composed of regions with different gray level ranges. The histogram of the image can be separated into a number of peaks, each corresponding to one region and there exists a threshold value corresponding to valley between the two adjacent peaks [1]. As for color images, the situation is different from monochrome image because of multi-features. Multiple histogram-based thresholding divides color space by thresholding each component histogram

Histograms are constructed by splitting the range of the data into equal-sized classes. Then for each class, the number of points from the data set that fall into each class is counted. Suppose that the gray-level histogram corresponds to an image $\mathrm{f}(\mathrm{x}, \mathrm{y})$ composed of dark objects on the light background, in such a way that object and background pixels have gray levels grouped into two dominant modes [7]. One obvious way to extract the objects from the background is to select a threshold ' $\mathrm{T}$ ' that separates these modes. Then any point $(\mathrm{x}, \mathrm{y})$ for which $\mathrm{f}(\mathrm{x}, \mathrm{y})<\mathrm{T}$ is called an object point, otherwise, the point is called a background point [7].

\section{Bimodal Histogram}

If two dominant modes characterize the image histogram, it is called a bimodal histogram. Only one threshold is enough for partitioning the image. If for example an image is composed of two types of dark objects on a light background, three or more dominant modes characterize the image histogram.

\section{Multimodal Histogram}

In such a case the histogram has to be partitioned by multiple thresholds. Multilevel thresholding classifies a point $(\mathrm{x}, \mathrm{y})$ as belonging to one object class if $\mathrm{T} 1<(\mathrm{x}, \mathrm{y}) \leq \mathrm{T} 2$, to the other object class if $\mathrm{f}(\mathrm{x}, \mathrm{y})>\mathrm{T} 2$ and to the background if $\mathrm{f}(\mathrm{x}$, $\mathrm{y}) \leq \mathrm{T} 1$.

Basic Global Thresholding includes:

\section{1) Select an initial estimate for $T$}

2) Segment the image using $T$. This will produce two groups of pixels. G1 consisting of all pixels with gray level values $>\mathrm{T}$ and $\mathrm{G} 2$ consisting of pixels with values $\leq \mathrm{T}$.

3) Compute the average gray level values mean 1 and mean 2 for the pixels in regions $\mathrm{G} 1$ and $\mathrm{G} 2$.

4) Compute a new threshold value

$$
\mathrm{T}=(1 / 2)(\text { mean } 1+\text { mean } 2)
$$

5) Repeat steps 2 through 4 until difference in $T$ in successive iterations is smaller than a predefined parameter T0. 


\section{Multilevel thresholding}

Effective thresholding can be achieved by a recursive multilevel thresholding method. In $1^{\text {st }}$ stage, threshold is set to separate brighter region from darker regions by locating minimum value between luminance modes of histogram. Then histograms are formed for each segmented parts. If these are not unimodal, for each part threshold is set again. This process is repeated till histogram of part becomes unimodal.

\section{Thresholding - Color Images}

In color images each pixel is characterized by three RGB values, intensity, hue and saturation. If the property histograms are all not unimodal, a procedure is invoked to determine best property and best level for thresholding of that property. Histograms are plotted for each of the color values and threshold points are found. Procedure is repeated on each part until resulting histogram becomes unimodal.

\section{OTSU method}

In this method, threshold can be directly computed without pre-treatment to histogram. This algorithm is simple and is a remarkable method for selecting the threshold. Here's the fundamental principle. The gray value of a grey-scale map is 0 to 255.The total number of pixels is defined as $N, i n$ is the number of pixels which's gray value is $i$. By normalizing the histogram, the following equations could be obtained.

$$
\begin{gathered}
\sum_{i=0}^{255} n_{i}=N \\
p_{i}=\frac{n_{i}}{N}
\end{gathered}
$$

$p_{i}$ is the probability of the pixels which's gray value is $i$. The threshold of the image segmentation is defined as $m$, then the probability $\theta_{0}$ and mean value $\mu_{0}$ of the background can be obtained through the following equations:

$$
\begin{gathered}
\theta_{0}=\sum_{i=0}^{m} p_{i} \\
\mu_{0}=\frac{\sum_{i=0}^{m} i p_{i}}{\theta_{0}}
\end{gathered}
$$

probability and typical value of the target also can be obtained:

$$
\begin{gathered}
\theta_{1}=\sum_{i=m+1}^{255} i p_{i} \\
\mu_{1}=\frac{\sum_{i=m+1}^{255} i p_{i}}{\theta_{1}}
\end{gathered}
$$

By computing all the above values, the following equation is obtained

$$
\sigma_{B}^{2}=\theta_{0} \theta_{1}\left(\mu_{0}-\mu_{1}\right)^{2}
$$

Variance is a metric of the uniformity of distribution, the greater the variance yields, the greater the difference between the target and the background. Therefore, the threshold which makes the variance yields maximal is the optimal threshold.

\section{B. Clustering}

K-Means Clustering Algorithm will be used as a clustering method. It is one of the simplest unsupervised learning algorithms that solve the well known clustering problem. The procedure follows a simple and easy way to classify a given data set through a certain number of clusters (assume $\mathrm{k}$ clusters) fixed a priori. The main idea is to define $\mathrm{k}$ centroids, one for each cluster. These centroids are placed at different location to cause different results. So, the better choice is to place them as much as possible far away from each other. The next step is to take each point belonging to a given data set and associate it to the nearest centroids [3]. When no point is pending, the first step is completed and an early grouping is done. At this point we need to re-calculate $\mathrm{k}$ new centroids as barycentre of the clusters resulting from the previous step. After we have these k new centroids, a new binding has to be done between the same data set points and the nearest new centroids. A loop has been generated. As a result of this loop we may notice that the $\mathrm{k}$ centroids change their location step by step until no more changes are done. In other words centroids do not move any more.

The K-Means algorithm is an iterative technique that is used to partition an image into $K$ clusters.

The basic algorithm is:

- $\quad$ Pick $K$ cluster centres, either randomly or based on some trial and error.

- Assign each pixel in the image to the cluster that minimizes the distance, pixel color difference, intensity, texture, and location between the pixel and the cluster centre

- Re-compute the cluster centres by averaging all of the pixels in the cluster

- Repeat steps 2 and 3 until convergence is attained (e.g. no pixels change clusters)

A drawback of the k-means algorithm is that the number of clusters $\mathrm{k}$ is an input parameter. An inappropriate choice of $\mathrm{k}$ may yield poor results.

\section{Region Growing}

Region growing is a procedure that groups pixels or sub regions into larger regions. The simplest of these approaches is pixel aggregation, which starts with a set of "seed" points and from these grows regions by appending to each seed points those neighboring pixels that have similar properties (such as gray level, texture, color, shape) [11][12].

The other procedure is Similarity Measures, in which individual pixel intensities are compared.

$>$ The other method is Comparing to Neighbour in Region. By this way, each pixel that is already in the region can bring in neighbours who are like it. 
$>$ The other method is Merging, in which adjacent similar pixels and similar regions are merged. Eventually, this method will converge when no further such merging are possible

\section{Split and Merge Technique}

- $\quad$ Pure merging methods are, however, computationally expensive because they start from such small initial regions (individual points) [12][13][15].

- $\quad$ First, we must split the image. Start by considering the entire image as one region.

1. If the entire region is coherent (i.e., if all pixels in the region have sufficient similarity), leave it unmodified.

2. If the region is not sufficiently coherent, split it into four quadrants and recursively apply these steps to each new region.

\section{Edge detection techniques}

Edge detection uses the difference in color between the background color and the foreground color. The end result is an outline of the borders. It is extensively used for gray level image segmentation, which is based on the detection of discontinuity in gray level, trying to locate points with abrupt changes in gray level. In principle, the edge detection operator can be applied simultaneously all over the image[6]. One technique is high-emphasis spatial frequency filtering. Since high spatial frequencies are associated with sharp changes in intensity, one can enhance or extract edges by performing high-pass filtering using the Fourier operator. The problem is how to design a relevant filter. In a monochrome image, edge is defined as a discontinuity in the gray level, and can be only detected when there is a difference of the brightness between two regions. There exist complex edge detectors that are also applied on color images[5][15].

\section{The types of edge detection techniques are}

\section{$>$ Gradient}

Detects the edges by looking for the maximum and minimum in the first derivative of the image

- Sobel

- Roberts

- Prewitt

$>$ Laplacian

The purpose of this method is extracting the intensity from edges

\section{$>$ Canny edge detection algorithm}

\section{Gradient Based Edge Detection:}

The assumption is edges are the pixels with a high gradient.These are implemented with convolution mask and

based on discrete approximations to differential operators. The quality of the edge image depends on the threshold. These are sensitive to noise and inaccurate.

\section{Laplacian operator:}

The Laplacian may be used when we are interested only in edge magnitudes without regard to their orientations. The Laplacian has the same properties in all directions and is therefore invariant to rotation in the image. The disadvantage is malfunctioning at the corners, curves and where the grey level intensity function varies. Not finding the orientation of edge because of using the Laplacian filter [15].

\section{Canny Edge Detector:}

Canny specified three issues that an edge detector must address. They are:-

1. Error rate:-The edge detector should respond only to edges, and should find all of them; no edges should be missed.

2. Localization:- The distance between the edge pixels as found by the edge detector and the actual edge should be as small as possible.

3. Response:- The edge detector should not identify multiple edge pixels where only a single edge exists.

Steps:

1. Apply derivative of Gaussian

2. Non-maximum suppression

- Thin multi-pixel wide "ridges" down to single pixel width

3. Linking and thresholding

- Low, high edge-strength thresholds

- Accept all edges over low threshold that are connected to edge over high threshold

To improve current methods of edge detectors we must follow 1. The first and most obvious is low error rate. It is important that edges occurring in images should not be missed and that there be no responses to non-edges.

2. The second criterion is that the edge points be well localized i.e., the distance between the edge pixels as found by the detector and the actual edge is to be at a minimum.

3. A third criterion is to have only one response to a single edge[15].

Many of the existing segmentation techniques, such as clustering methods in colour space work well on homogeneous colour regions. Natural scenes are rich in colour and texture. Many texture segmentation algorithms require the estimation of texture model parameters.

Parameter estimation is a difficult problem and often requires a good homogeneous region for robust estimation.

\section{E. Watershed algorithm}

Watersheds are one of the classic regions in the field of topography. A drop of the water falling it flows down until it reaches the button of the region. Monochrome image is considered to be an altitude surface in which high-altitude pixels correspond to ridges and low-altitude pixels correspond to valleys [2] [8]. This idea says if we have a minima point, by falling water, region and the boundary can be achieved. Watershed use image gradient to initial point and region can obtain by region growing. The accumulation of water in the vicinity of local minima is called a catchment basin[14][15]. 
The term watershed refers to a ridge that divides areas drained by different river systems. A catchment basin is the geographical area draining into a river or reservoir. If you imagine that bright areas are "high" and dark areas are "low," then it might look like the surface (left). With surfaces, it is natural to think in terms of catchment basins and watershed lines[8].

There are two approaches to find watershed of an image

i. Rainfall approach

ii. Flooding approach

In rainfall approach, local minima are found throughout the image, and each local minima is assigned unique tag. A conceptual water drop is placed at each untagged pixel. The drop moves to low-amplitude neighbor until it reaches a tagged pixel and it assumes tag value[14].

In flooding approach, conceptual pixel holes are pierced at each local minima. The water enters the holes and proceeds to fill each catchment basin. If the basin is about to overflow, a dam is built on its surrounding ridge line to the height of high altitude ridge point. These dam boundaries correspond to the watershed lines[14].

In the first step wavelet transform for producing approximation and detail images is applied, then by Sobel mask, approximation image gradient is obtained and additional edge is eliminated by a threshold then watershed transform for obtaining initial segmentation is applied and segmented image is projected to high resolution by inverse wavelet using segmented image in low resolution and detail images are updated. Region merging is applied in the last.

Steps in Watershed Algorithm:

- Read in an Image and covert it in grayscale

- Use the gradient magnitude as the segmentation function

- Mark the foreground objects

- Compute the Background markers

- Compute the watershed transform of the segmentation function

- Visualize the result.

The main problem of this algorithm is over segmentation, because all of edge and noise would appear in the image gradient, which make the denoising process necessary.

\section{F. Level-set method}

The level set method was initially proposed to track moving and can be used to efficiently address the problem of curve/surface/etc. The central idea is to represent the evolving contour using a signed function, where its zero level corresponds to the actual contour. Then, according to the motion equation of the contour, one can easily derive a similar flow for the implicit surface that when applied to the zerolevel will reflect the propagation of the contour. The level set method encodes numerous advantages: it is implicit, parameter free, provides a direct way to estimate the geometric properties of the evolving structure, can change the topology and is intrinsic.

\section{G. Texture based segmentation}

In case of images wherein all of the boundaries arise from neighbouring patches of different texture, we compute pairwise texture similarities by comparing windowed texton histograms. The $\chi 2$ test is performed to test the histograms which is defined as

$$
\chi^{2}\left(h_{i,} h_{j}\right)=\frac{1}{2} \sum_{k=1}^{K} \frac{\left[h_{i}(k)-h_{j}(k)\right]^{2}}{h_{i}(k)+h_{j}(k)}
$$

The weight is calculated using the above equation.

The procedure consists of the following 4 steps:

1. Compute an initial segmentation from the locally estimated weight matrix.

2. Update the weights using the initial segmentation.

3. Coarsen the graph with the updated weights to reduce the segmentation to a much simpler problem.

4. Compute a final segmentation using the coarsened graph.

A drawback is, texture is measured over a window area and so texture measurements in vicinity of boundary between texture regions represent some average texture computation. So it becomes difficult to locate the boundary [9].

\section{H. Wavelet based segmentation}

The wavelet transform is important to provide a compact description of signals (or images) that are limited in time (spatial extent) and it is very helpful in description of edge and line that are highly localized [5].

2-D wavelet decomposition is use for images. This 2-D wavelet transform requires two wavelets, namely, $\psi_{1}(x, y)$ and $\psi_{2}(x, y)$. At a particular scale s we have:

$$
\psi_{s}^{i}(x, y)=\frac{1}{s^{2}} \psi^{i}\left(\frac{x}{s}, \frac{y}{s}\right) \quad i=1,2
$$

By applying each one $f(x, y)$, at a scale $s=2 j$ we will have a component

$$
w_{2^{j}}^{i} f(x, y)=\left(f * w_{2^{j}}^{i}\right)(x, y) i=1,2
$$

Then the original signal $f(x, y)$ can be represented by the 2-D wavelet transform, in terms of the two dual wavelets $\xi^{1}(x, y)$ and $\xi^{2}(x, y)$

$$
f(x, y)=\varepsilon\left(\left(W_{2^{j}}^{1} f * \xi_{2^{j}}^{1}\right)(x, y)+\left(w_{2^{j}}^{2} f * \xi_{2^{j}}^{2}\right)(x, y)\right.
$$

And it is required a scaling function $\varphi(x, y)$ for build a multistage representation. Corresponding component at a scale $2 j$ is:

$$
S_{2^{j}} f(x, y)=\left(f * \phi_{2^{j}}\right)(x, y)
$$

These wavelet measure functions are variations along different directions. We may interpret the component $S_{2^{j}} f(x, y)$ as a smoothed version of $f(x, y)$, and the components, for $j=1, \ldots, J$, as the image details lost by smoothing going from $S_{2^{0}} f(x, y)$ to $S_{2^{1}} f(x, y)$. 
Indeed, by using wavelet on an image for one level, four images will be obtained which correspond to the approximation and detail images.

\section{Genetic algorithm (GA) based segmentation}

Genetic algorithm which imitates the principles of biological evolution is a method for global search and optimization. GA begins at a random initial group and then adopts selection, crossover and mutation, and some other genetic operations based on the principle of survival of the fittest to obtain a preferable group. The algorithm keeps iterating until meets the terminal condition [10] [15].

The steps in the algorithm are

1. Initialize segments in the primary regions and define correspondence between each region and related position of its label in the code strings generated by a genetic algorithm.

2. Construct primary region adjacency graph.

3. Pick starting population at random. If priory information is available, that can help to define the population.

4. Genetic optimization: Collapse region adjacency graph for each code string of current population. Using current region adjacency graph, compute the value of optimization segmentation function for each code string from population.

5. If maximum of optimum criterion does not increase significantly in several consecutive steps, go to step 7.

6. Let genetic algorithm generate new population of segmentation hypothesis.

7. The code string with maximum confidence (best hypothesis) represents final image segmentation

\section{J. Wavelength based approach}

Wavelengths of the color images ranges from 760-380nm.In proposed algorithm the ranges of R G B components are prespecified. Each component of the color is considered and based on their color value, if it is within pre-specified value then that color is matched and that component is displayed. For the basic colors, that particular color component ranges from 0-255 and other two components will be half of that value and for the secondary colors the combination of the component colors are considered.

\section{COMPARISION PARAMETERS}

In this paper the comparison of the segmented images are done by taking the entropy and mutual information measures. The entropy of an image can be defined as a measure of the uncertainty associated with a random variable and it quantifies, in the sense of an expected value, the information contained in a message. Entropy of an image E returns a scalar value representing the entropy of grayscale image I. Entropy is a statistical measure of randomness that can be used to characterize the texture of the input image. Entropy is defined as -sum $\left(p .{ }^{*} \log _{2}(p)\right)$ where $p$ contains the histogram counts.

Mutual information of two random variables is a quantity that measures the mutual dependence of the two variables. For two discrete variables $\mathrm{X}$ and $\mathrm{Y}$ whose joint probability is given
$\mathrm{P}_{\mathrm{XY}}(\mathrm{x}, \mathrm{y})$, the mutual information between them, denoted $\mathrm{I}(\mathrm{X} ; \mathrm{Y})$, is given by

$$
I(X ; Y)=\sum_{x, y} P_{X Y}(x, y) \log \frac{P_{X Y}(x, y)}{P_{X}(x) P_{Y}(y)}
$$

Here $\mathrm{P}_{\mathrm{X}}$ and $\mathrm{P}_{\mathrm{Y}}$ are the marginal values:

$$
\begin{aligned}
& P_{X}(x)=\sum_{y} P_{X Y} \log \frac{P_{X Y}}{P_{X} P_{Y}} \text { and } \\
& P_{Y}(y)=\sum_{x} P_{X Y}(X, Y)
\end{aligned}
$$

\section{IMPLEMENTATION}

The above discussed algorithms are implemented using MATLAB 7.4.0(R2009a). The experiments are conducted at PESIT Telecommunication R\&D Centre for various set of input images and image formats. The different set of input medical images such as X-ray, MRI and CT are considered for experimentation. Also the different image file formats such as JPEG, TIFF, BMP, GIF, text and PNG are considered. The sample results are displayed for the discussion.

\section{RESULTS}

The segmentation algorithms are applied to various formats of images and few samples are given below.

TABLE 1: ENTROPY \& MUTUAL INFORMATION (MI) OF

\begin{tabular}{|c|c|c|}
\hline \multicolumn{3}{|c|}{ BABY.JPG IMAGE } \\
\hline Original image & $\begin{array}{c}\text { Mutual information } \\
\text { (MI) With respect to } \\
\text { original image }\end{array}$ & Entropy \\
\hline Texture based & 7.7123 & 7.7123 \\
\hline Genetic algorithm & 0.1130 & 6.2662 \\
\hline
\end{tabular}

TABLE 2: ENTROPY \& MUTUAL INFORMATION (MI) OF EDGE DETECTORS

\begin{tabular}{|c|c|c|}
\hline Edge detectors & MI & Entropy \\
\hline Canny & 0.0287 & 2.4095 \\
\hline Sobel & 0.0302 & 1.7232 \\
\hline Roberts & 0.0310 & 1.5261 \\
\hline Prewitt & 0.0308 & 1.6631 \\
\hline LOG & 0.0304 & 2.0870 \\
\hline
\end{tabular}

TABLE 3: ENTROPY \& MUTUAL INFORMATION (MI)

\begin{tabular}{|c|c|c|}
\hline \multicolumn{3}{c}{ CLUSTERING } \\
\hline $\begin{array}{c}\text { Klustering } \\
\text { K-Means } \\
\text { algorithm cluster1 }\end{array}$ & 0.1369 & Entropy \\
\hline $\begin{array}{c}\text { K-means } \\
\text { algorithm cluster2 }\end{array}$ & 0.1435 & 3.6174 \\
\hline
\end{tabular}

TABLE 4: ENTROPY \& MUTUAL INFORMATION (MI) OF REGION

\begin{tabular}{|c|c|c|}
\multicolumn{3}{|c|}{ BASED TECHNIQUES } \\
\hline Region Based & MI & Entropy \\
\hline $\begin{array}{c}\text { Contour based } \\
\text { segmentation }\end{array}$ & 0.1648 & 6.0032 \\
\hline $\begin{array}{c}\text { Contour based } \\
\text { with noise }\end{array}$ & 0.2638 & 1.5237 \\
\hline
\end{tabular}


H.S.Prasantha et. al. / (IJCSE) International Journal on Computer Science and Engineering Vol. 02, No. 04, 2010, 1209-1218

\begin{tabular}{|c|c|c|}
\hline Chenvese method & 0.1913 & 5.6762 \\
\hline Region grow & 0.1216 & 1.4511 \\
\hline
\end{tabular}

TABLE 5: ENTROPY \& MUTUAL INFORMATION (MI) OF WAVELETS TECHNIQUES

\begin{tabular}{|c|c|c|}
\hline Wavelets & MI & Entropy \\
\hline $\begin{array}{c}\text { Wavelet based- } \\
\text { Haar }\end{array}$ & 0.1114 & 3.4983 \\
\hline $\begin{array}{c}\text { Wavelet based- } \\
\text { db8 }\end{array}$ & 0.1091 & 3.0246 \\
\hline $\begin{array}{c}\text { Wavelet based- } \\
\text { db16 }\end{array}$ & 0.1097 & 3.114 \\
\hline
\end{tabular}

TABLE 6:ENTROPY \& MUTUAL INFORMATION (MI) OF THRESHOLD BASED TECHNIQUES

\begin{tabular}{|c|c|c|}
\hline Threshold & MI & Entropy \\
\hline $\begin{array}{c}\text { Thresholding } \\
0.2\end{array}$ & 0.7618 & 1.2383 \\
\hline $\begin{array}{c}\text { Thresholding } \\
0.5\end{array}$ & 0.0269 & 1.6642 \\
\hline $\begin{array}{c}\text { Thresholding } \\
0.9\end{array}$ & 0.0330 & 1.7033 \\
\hline Local threshold & 0.0304 & 1.5697 \\
\hline Iterative threshold & 0.0259 & 1.7033 \\
\hline Otsu method & 0.0249 & 1.685 \\
\hline $\begin{array}{c}\text { Adaptive } \\
\text { Thresholding }\end{array}$ & 0.0263 & 0.3937 \\
\hline
\end{tabular}

TABLE 7: ENTROPY \& MUTUAL INFORMATION (MI) OF WATERSHED BASED TECHNIQUES

\begin{tabular}{|c|c|c|}
\hline Watershed & MI & Entropy \\
\hline $\begin{array}{c}\text { Watershed } \\
\text { algorithm }\end{array}$ & 0.1556 & 2.5733 \\
\hline $\begin{array}{c}\text { Marker controlled } \\
\text { watershed }\end{array}$ & 0.3372 & 3.4652 \\
\hline
\end{tabular}

TABLE 8

ENTROPY VALUES OF BIOMEDICAL IMAGES

\begin{tabular}{|c|c|c|c|}
\hline Original & XRAY & CT & MRI \\
\hline Original & 7.53363 & 7.51363 & 6.32155 \\
\hline Genetic & 0.99963 & 0.99497 & 0.92476 \\
algorithm & 0.99938 & 0.99926 & 0.93478 \\
& & & \\
\hline Texture based & 5.4619 & 3.5541 & 3.5142 \\
& 3.4652 & 5.3501 & 3.7434 \\
\hline
\end{tabular}

TABLE 9

ENTROPY OF EDGE BASED TECHNIQUES

\begin{tabular}{|c|c|c|c|}
\hline $\begin{array}{c}\text { Edge } \\
\text { Detectors }\end{array}$ & X RAY & CT & MRI \\
\hline Canny & 0.80035 & 0.93575 & 0.86841 \\
\hline Sobel & 0.44565 & 0.44875 & 0.50653 \\
\hline Robert & 0.12120 & 0.15221 & 0.16882 \\
\hline Prewitt & 0.22506 & 0.15115 & 0.24267 \\
\hline LOG & 0.38254 & 0.35990 & 0.40764 \\
\hline
\end{tabular}

TABLE 10

ENTROPY OF THRESHOLD BASED TECHNIQUES

\begin{tabular}{|c|c|c|c|}
\hline Threshold & XRAY & CT & MRI \\
\hline $\begin{array}{c}\text { Adaptive } \\
\text { thresholding }\end{array}$ & 0.52999 & 0.80058 & 0.95452 \\
\hline $\begin{array}{c}\text { Iterative } \\
\text { thresholding }\end{array}$ & 0.0214 & 0.0207 & 0.0319 \\
\hline Otsu method & 0.0286 & 0.0236 & 0.0289 \\
\hline Local threshold & 0.0389 & 0.0300 & 0.0417 \\
\hline
\end{tabular}

TABLE 11

ENTROPY OF CLUSTERING BASED TECHNIQUES

\begin{tabular}{|c|c|c|c|}
\hline Clustering & XRAY & CT SCAN & MRI \\
\hline K means Cluster1 & 4.66585 & 4.24384 & 3.54521 \\
\hline Cluster2 & 3.93989 & 4.25797 & 3.75962 \\
\hline
\end{tabular}

TABLE 12

ENTROPY OF REGION BASED TECHNIQUES

\begin{tabular}{|c|c|c|c|}
\hline Region Based & X RAY & CT SCAN & MRI \\
\hline Region Grow & 6.81652 & 6.84531 & 6.33251 \\
\hline $\begin{array}{c}\text { Contour based } \\
\text { with added noise }\end{array}$ & 0.9997 & 0.9943 & 0.9400 \\
\hline Contour based & 0.95484 & 0.97086 & 0.93488 \\
\hline
\end{tabular}

TABLE 13

ENTROPY WATERSHEDBASED TECHNIQUES

\begin{tabular}{|c|c|c|c|}
\hline Watershed & XRAY & CT SCAN & MRI \\
\hline Watershed & 0.96496 & 0.9961 & 0.9991 \\
technique & 0.05332 & 0.1365 & 0.0321 \\
\hline $\begin{array}{c}\text { Marker controlled } \\
\text { Watershed }\end{array}$ & 0.99735 & 0.96981 & 0.99976 \\
\hline
\end{tabular}

TABLE 14

ENTROPY OF WAVELETS BASED TECHNIQUES

\begin{tabular}{|c|c|c|c|}
\hline Wavelets & XRAY & CT SCAN & MRI \\
\hline Haar & 0 & 0.00607 & 0 \\
& 1.26906 & 1.21786 & 1.31892 \\
& 1.31082 & 1.25087 & 1.33554 \\
& 1.38045 & 1.46152 & 1.37454 \\
\hline Db16 & 0.05000 & 0 & 0.55467 \\
& 2.71170 & 3.69464 & 2.14036 \\
& 2.76674 & 3.93040 & 2.36640 \\
& 3.84807 & 4.39078 & 2.85021 \\
\hline
\end{tabular}

TABLE 15

MUTUAL INFORMATION (MI) OF BIOMEDICAL IMAGES

\begin{tabular}{|c|c|c|c|}
\hline Original & XRAY & CT SCAN & MRI \\
\hline Genetic algorithm & 0.0321 & 0.0202 & 0.0534 \\
\hline Texture based & 0.1187 & 0.1076 & 0.1286 \\
\hline
\end{tabular}

TABLE 16

MUTUAL INFORMATION (MI) OF EDGE BASED TECHNIQUES

\begin{tabular}{|c|c|c|c|}
\hline $\begin{array}{c}\text { Edge } \\
\text { Detectors }\end{array}$ & XRAY & CT SCAN & MRI \\
\hline Canny & 0.0681 & 0.0364 & 0.0983 \\
\hline Sobel & 0.0211 & 0.0134 & 0.0573 \\
\hline Robert & 0.0066 & 0.0011 & 0.1001 \\
\hline Prewitt & 0.0321 & 0.0208 & 0.0632 \\
\hline LOG & 0.0548 & 0.0245 & 0.0944 \\
\hline
\end{tabular}

TABLE 17

MUTUAL INFORMATION (MI) OF THRESHOLD BASED TECHNIQUES

\begin{tabular}{|c|c|c|c|}
\hline Threshold & XRAY & CT SCAN & MRI \\
\hline Adaptive & 0.1233 & 0.0987 & 0.7346 \\
\hline Iterative & 0.0214 & 0.0207 & 0.0319 \\
\hline
\end{tabular}




\begin{tabular}{|c|c|c|c|}
\hline thresholding & & & \\
\hline Otsu method & 0.0286 & 0.0236 & 0.0289 \\
\hline Local threshold & 0.0389 & 0.0300 & 0.0417 \\
\hline
\end{tabular}

TABLE 18

MUTUAL INFORMATION (MI) OF CLUSTERING

\begin{tabular}{|c|c|c|c|}
\hline Clustering & XRAY & CTSCAN & MRI \\
\hline Kmeans cluster1 & 2.9226 & 2.4567 & 2.8928 \\
& & & 3.5029 \\
\hline Cluster2 & 4.2745 & 2.1064 & 3.5029 \\
\hline
\end{tabular}

TABLE 19

MUTUAL INFORMATION (MI) OF WATERSHED TECHNIQUES

\begin{tabular}{|c|c|c|c|}
\hline Watershed & XRAY & CT SCAN & MRI \\
\hline Watershed & 0.1567 & 0.1279 & 0.1879 \\
\hline $\begin{array}{c}\text { Marker controlled } \\
\text { Watershed }\end{array}$ & 0.3324 & 0.2987 & 0.3487 \\
\hline
\end{tabular}

TABLE 20

MUTUAL INFORMATION (MI) OF WAVELETS TECHNIQUES

\begin{tabular}{|c|c|c|c|}
\hline Wavelets & XRAY & CT SCAN & MRI \\
\hline Haar & 0.0012 & 0.0003 & 0.0021 \\
& 0.0165 & 0.0139 & 0.0189 \\
& 0.1276 & 0.0120 & 0.1678 \\
& 0.1314 & 0.1001 & 0.1887 \\
\hline Db16 & 0.002 & 0.0016 & 0.0028 \\
& 0.0236 & 0.0198 & 0.0367 \\
& 0.1878 & 0.1453 & 0.2764 \\
& 0.2325 & 0.1954 & 0.3475 \\
\hline
\end{tabular}

\section{CONCLUSION}

The image segmentation is a relevant technique in image processing. Numerous and varied methods exist for many applications. Now that we have described the algorithms, we can compare the outputs and check which type of segmentation technique is better for a particular format. It is believed that there are two key factors which allow for the use of a segmentation algorithm in a larger object detection system: correctness and stability.

On an average parameter set of the edge detection techniques, The canny operator performed better than all other operators for all the formats of images, sample of which can be found in results. The same is reflected by higher value of entropy after segmentation.

Histogram based methods are found to be very efficient in terms of computation complexity when compared to other image segmentation methods. If significant peaks and valleys are identified properly and proper thresholding is fixed, this technique yields good result. The same is reflected with a lower value of computation time.

Region-grow technique operates well over all formats of images provided proper seed point is selected and range of threshold is properly defined. This method performs well even when noise is present and it is reflected with a reasonably good value of entropy.

The clustering algorithm is guaranteed to converge but it may not return optimal solution. The quality of the solution depends on the initial set of clusters and value of $k$. An inappropriate choice of $\mathrm{K}$ yields very poor result. This algorithm can be directly applied for color images.

In watershed algorithms, the length of gradients is interpreted as elevation information. The flooding process is performed over gradient image, normally this leads to an over segmentation of an image, especially for noisy image material.

\section{REFERENCES}

[1] Deshmukh K.S. and Shinde G. N."An adaptive color image segmentation". Electronic Letters on Computer Vision and Image Analysis 5(4):12-23, 2005.

[2] Leila Shafarenko, Maria Petrou, and Josef Kittler. "Automatic Watershed Segmentation of Randomly Textured Color Images". IEEE transactions on image processing, vol.6, no.11, November 1997

[3]H.Essaqote, N.Zahid, I.Haddaoui and A.Ettouhami. "Color Image Segmentation Based On New Clustering Algorithm and Fuzzy Eigenspace”. Research Journal of Applied Sciences 2(8):853-858, 2007

[4] Alexandra Oliveros, and Miguel Sotaquirá." An Automatic Gridding and Contour Based Segmentation Approach Applied to DNA Microarray Image Analysis", International Journal of Biological and Life Sciences 3:4 2007.

[5] Jun Li." A Wavelet Approach to Edge Detection". MSc Thesis, Sam Houston State University Huntsville, Texas August, 2003.

[6] Wei-Ying Ma, and B. S. Manjunath. "Edge Flow: A Technique for Boundary Detection and Image Segmentation”. IEEE transactions on image processing, vol. 9, no. 8, august 2000.

[7] L.Shafarenko, M. Petrou, and J. Kittler. "Histogram-Based Segmentation in a Perceptually Uniform Color Space". IEEE transactions on image processing, vol. 7, no. 9, September 1998.

[8] Malik Sikandar Hayat Khiyal, Aihab Khan, and Amna Bibi. "Modified Watershed Algorithm for Segmentation of $2 D$ Images". Issues in Informing Science and Information Technology Volume 6, 2009.

[9] B. B. Chaudhuri and Nirupam Sarkar. "Texture Segmentation Using Fractal Dimension". IEEE transactions on pattern analysis and machine intelligence, vol. 17, no. 1, January 1995.

[10] Lei Hui, Cheng Shi, Ao Min-si, Wu Yi-qi "Application of an Improved Genetic Algorithm in Image Segmentation”. 2008 International Conference on Computer Science and Software Engineering.

[11] Dr.S.V.Kasmir Raja, A.Shaik Abdul Khadir, Dr.S.S.Riaz Ahamed." Moving Toward Region-Based Image Segmentation Techniques: A Study" .Journal of Theoretical and Applied Information Technology 2005 - 2009 JATIT.

[12] S. A. Hojjatoleslami and J. Kittler." Region Growing: A New Approach”. IEEE Transactions On Image Processing, Vol. 7, No. 7, July 1998.

[13] Luis Garcia Ugarriza, Eli Saber, Senior, Sreenath Rao Vantaram, Vincent Amuso, Mark Shaw, and Ranjit Bhaskar. "Automatic Image Segmentation by Dynamic Region Growth and Multiresolution Merging”. IEEE Transactions on Image Processing, Vol. 18, No. 10, October 2009

[14] Rafael C. Gonzalez, Richard E. Woods." Digital Image processing ". $2^{\text {nd }}$ edition, Pearson Education,pp.589-656(2007)

[15] Milan Sonka, Vaclav Hlavac, Roger Boyle. "Image Processing, Analysis, and Machine Vision”, $2^{\text {nd }}$ edition, Thomas Learning, pp.123-190 (1998) 

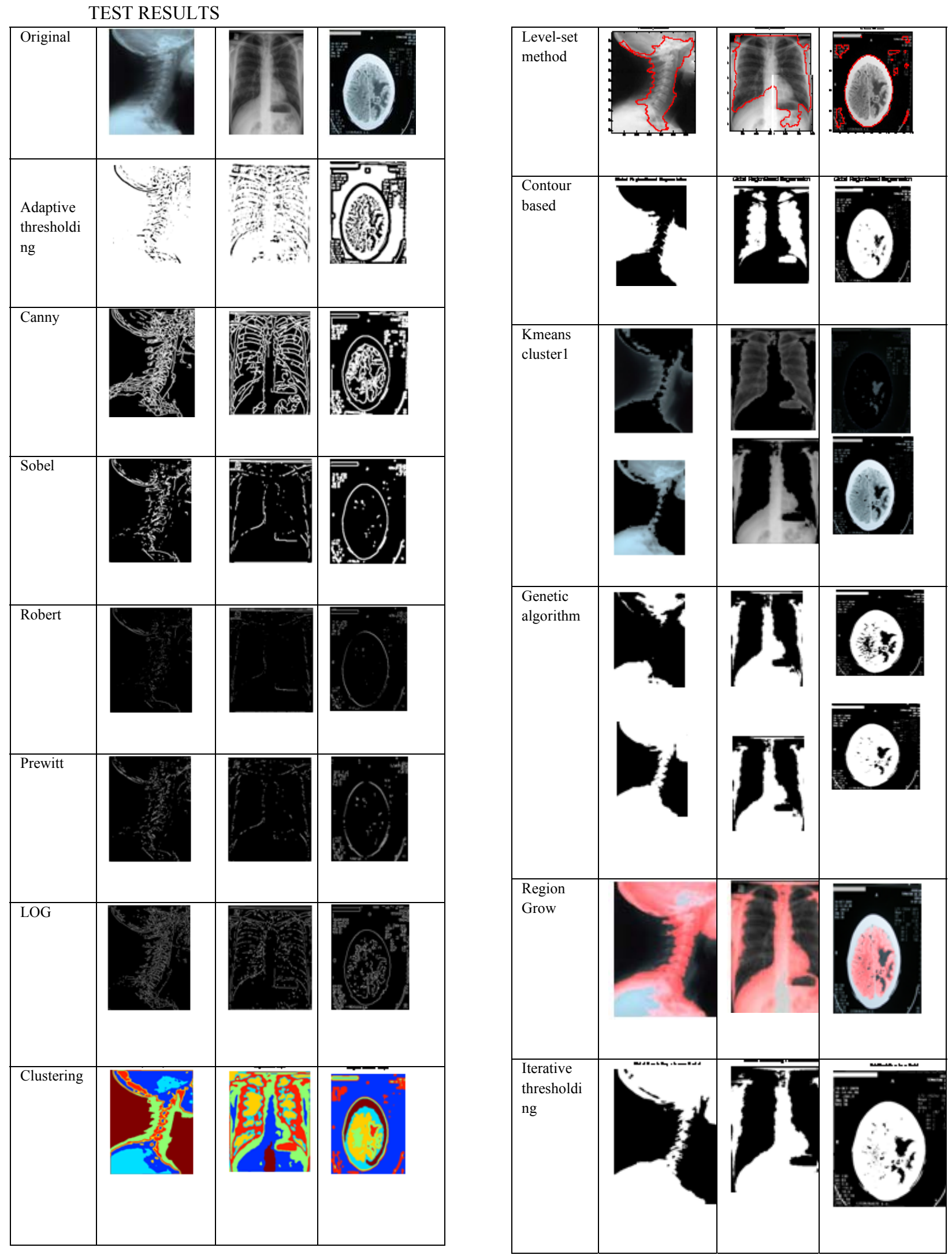
H.S.Prasantha et. al. / (IJCSE) International Journal on Computer Science and Engineering Vol. 02, No. 04, 2010, 1209-1218
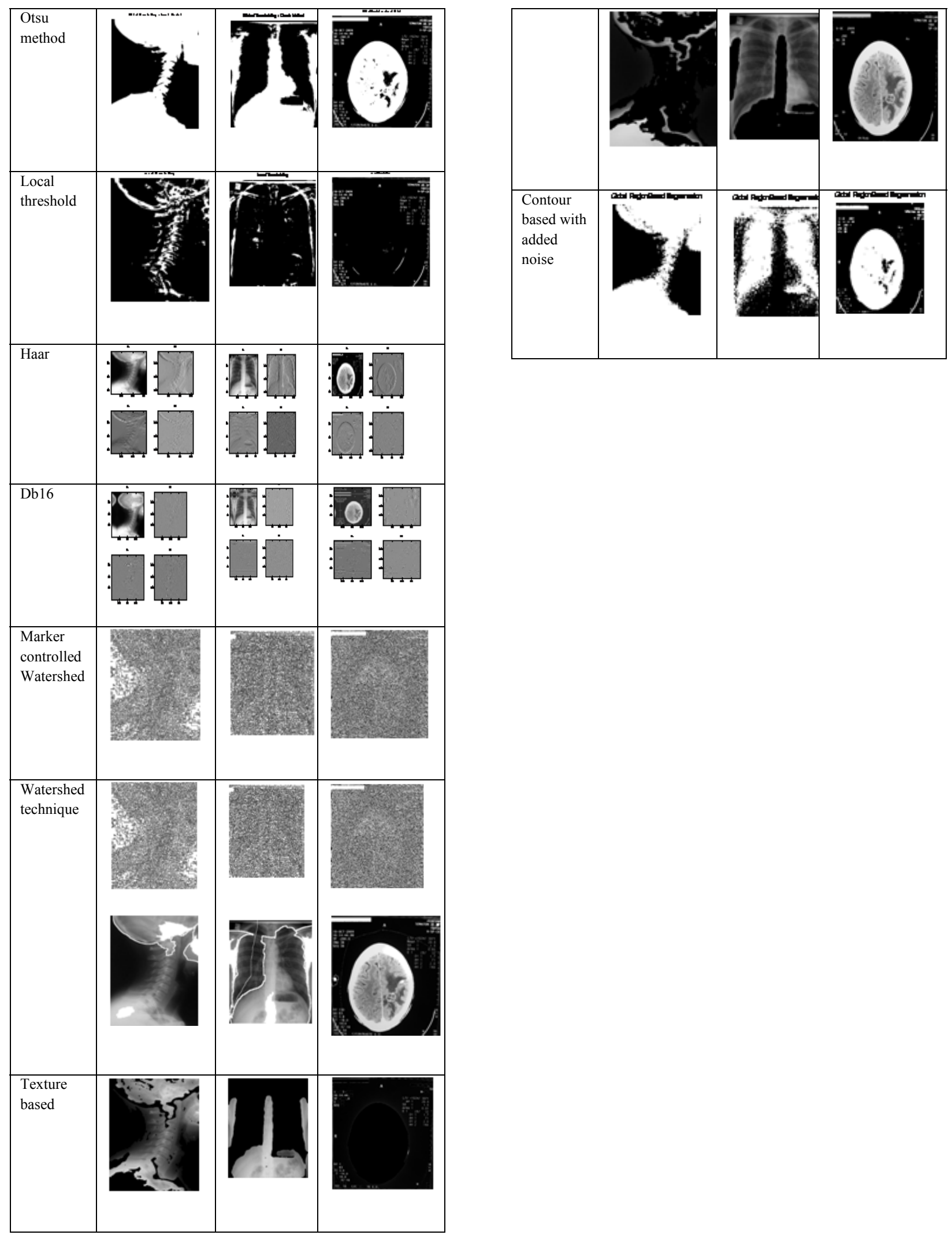


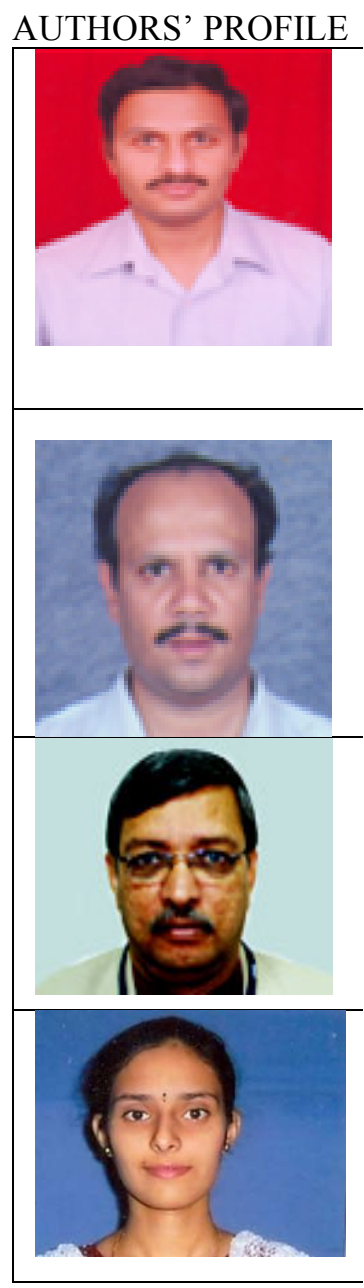

H.S.Prashantha received Bachelor degree from Bangalore University and Master Degree from V.T.U, Belgaum. Currently he is pursuing his PhD from Anna University, Coimbatore in the area of Image Processing. His research interest includes Digital Image Processing and Digital Signal Processing. Currently he is working as Assistant Professor in the department of Telecommunication Engineering at PES Institute of Technology, Bangalore.

Dr. Shashidhara.H.L received Bachelor degree from Bangalore University, Master degree from Mysore University and $\mathrm{PhD}$ from IIT, Bombay. His research interest includes Digital Signal Processing, Digital Image Processing, Artificial Neural Networks and Distributes systems. Currently he is working as Professor \& KRP in the department of Telecommunication Engineering at PES Institute of Technology, Bangalore.

Dr.K.N.B.Murthy received Bachelor degree from Mysore University, Master Degree from IISC, Bangalore and PhD from IIT, Madras. His research interest includes Digital Image Processing, Parallel Processing, etc. Currently he is working as Principal \& Director at PES Institute of Technology, Bangalore.

Madhavi lata.G received her Bachelor degree from VTU and currently pursuing her Master degree from VTU, Belgaum. Her research interest includes Image Processing and VLSI design. 